\title{
The Space of Morphisms on Projective Space
}

\author{
Alon Levy \\ November 15, 2018
}

\begin{abstract}
The theory of moduli of morphisms on $\mathbb{P}^{n}$ generalizes the study of rational maps on $\mathbb{P}^{1}$. This paper proves three results about the space of morphisms on $\mathbb{P}^{n}$ of degree $d>1$, and its quotient by the conjugation action of $\operatorname{PGL}(n+1)$. First, we prove that this quotient is geometric, and compute the stable and semistable completions of the space of morphisms. This strengthens previous results of Silverman, as well as of Petsche, Szpiro, and Tepper. Second, we bound the size of the stabilizer group in $\operatorname{PGL}(n+1)$ of every morphism in terms of only $n$ and $d$. Third, we specialize to the case where $n=1$, and show that the quotient space is rational for all $d>1$; this partly generalizes a result of Silverman about the case $d=2$.
\end{abstract}

\section{Introduction and Notation}

A rational map from $\mathbb{P}^{n}$ to itself is determined by an $(n+1)$-tuple of polynomials in $n+1$ variables, all homogeneous of the same degree $d$. If this map is a morphism, it will be finite of degree $d^{n}$. In the rest of this paper, we will refer to such a rational map as a degree $d$ map on $\mathbb{P}^{n}$ by abuse of notation. The space of degree $d$ maps on $\mathbb{P}^{n}$ is projective, with homogeneous coordinates coming from monomials of degree $d$. There are $\left(\begin{array}{c}n+d \\ d\end{array}\right)$ such monomials, so that this space has dimension $\left(\begin{array}{c}n+d \\ d\end{array}\right)(n+1)-1$. We write $N_{d}^{n}$ for the dimension of this space, or $N$ when $d$ and $n$ are clear.

The case of interest is morphisms on $\mathbb{P}^{n}$. In the sequel, we refer to the polynomials defining the map as $q_{0}, q_{1}, \ldots, q_{n}$. Then a map $\left(q_{0}: \ldots: q_{n}\right)$ is a morphism if and only if the $q_{i}$ 's share no common geometric root. The $q_{i}$ 's only share a common root on a hypersurface of $\mathbb{P}^{N}$ which we call the resultant subvariety and which is defined over $\mathbb{Z}$; we denote its complement by $\operatorname{Hom}_{d}^{n}$.

The space $\mathbb{P}^{N}$ of rational maps comes equipped with an action of $\operatorname{PGL}(n+1)$ by conjugation. The conjugation action $A \cdot \varphi=A \varphi A^{-1}$, fixes the resultant, which gives an action of $\operatorname{PGL}(n+1)$ on $\operatorname{Hom}_{d}^{n}$. In this paper, we mainly study the quotient of this action, which we denote $\mathrm{M}_{d}^{n}$, or $\mathrm{M}_{d}$ when $n=1$. We will show that this quotient is geometric in the sense of geometric invariant theory [8], and compute the largest stable and semistable loci $\operatorname{Hom}_{d}^{n, s}$ and $\operatorname{Hom}_{d}^{n, s s}$, which satisfy $\operatorname{Hom}_{d}^{n} \subset \operatorname{Hom}_{d}^{n, s} \subset \operatorname{Hom}_{d}^{n, s s} \subset$ $\mathbb{P}^{N}$. 
Knowing that the quotient $\mathrm{M}_{d}^{n}$ is well-behaved is often necessary to answer questions about the geometry of families of dynamical systems. In [10, Petsche, Szpiro, and Tepper prove that $\mathrm{M}_{d}^{n}$ exists as a geometric quotient in order to show that isotriviality is equivalent to potential good reduction for morphisms of $\mathbb{P}^{n}$ over function fields, generalizing previous results in the one-dimensional case. In [3], DeMarco uses the explicit description of the space $\mathrm{M}_{2}$ in order to study iterations of quadratic maps on $\mathbb{P}^{1}$, and one can expect similar results in higher dimension given a better understanding of the structure of $\mathrm{M}_{d}^{n}$.

By now the theory of morphisms on $\mathbb{P}^{1}$ is the standard example in dynamical systems. For a survey of the arithmetic theory, see [14]; also see a recent paper by Manes [5] about moduli of morphisms on $\mathbb{P}^{1}$ with a marked point of period $n$, which functions as a dynamical level structure. In the complex case, see an overview by Milnor [7], and the work of DeMarco [2] 3] about compactifications of the space $\mathrm{M}_{d}$ that respect the iteration map. Despite this, the higher-dimensional theory remains understudied. The only prior result in the direction of moduli of morphisms on $\mathbb{P}^{n}$ is the proof in [10] that $\mathrm{M}_{d}^{n}$ exists as a geometric quotient. Unfortunately, the proof does not lend itself well to finding the stable and semistable spaces for the action of $\operatorname{PGL}(n+1)$ on $\mathbb{P}^{N}$, nor does it bound the size of the finite stabilizer group uniformly on $\operatorname{Hom}_{d}^{n}$.

The first two tasks in this paper are then to construct alternative proofs of the fact that the quotient $\mathrm{M}_{d}^{n}$ is geometric, first by explicitly describing the stable and semistable loci, and second by finding a uniform bound for the size of the stabilizer group in $\operatorname{PGL}(n+1)$. The former we will do in section 2, using the Hilbert-Mumford criterion for stability and semistability. We will see that the complements of both $\operatorname{Hom}_{d}^{n, s}$ and $\operatorname{Hom}_{d}^{n, s s}$ are equal to a finite union of linear subvarieties and their PGL $(n+$ 1)-conjugates; this contrasts with the $n=1$ case, when the complement is the PGL(2)orbit of only one linear subvariety. In section 3 we will study the stabilizer groups, proving a uniform bound on their sizes, valid over all fields and rings of definition, depending only on $n$ and $d$. This will strengthen previous results in this direction for $n=1$ in $[12$.

Most results in this paper are a natural generalization of the study of morphisms on $\mathbb{P}^{1}$ in [13], which refers to the space of morphisms as Rat ${ }_{d}$ and its quotient as $\mathrm{M}_{d}$, and which proves that $\mathrm{M}_{2} \cong_{\text {Spec } \mathbb{Z}} \mathbb{A}^{2}$ using the theories of fixed points and multipliers. Specializing to the case where $n=1$, we will prove that $\mathrm{M}_{d}$ is rational for all $d$ in section 4. This is new even in the case of $d=3$. The proof in this paper is based on showing that $\mathrm{M}_{d}$ is birational to a vector bundle over the space $\mathrm{M}_{0, d+1}$ of $d+1$ unmarked points on $\mathbb{P}^{1}$, which is known to be rational.

Unfortunately, we do not see any easy generalization of rationality to $\mathrm{M}_{d}^{n}$. The obstruction is that the space of unmarked points on $\mathbb{P}^{n}$ is not known to be rational. Clearly $\operatorname{Hom}_{d}^{n}$ is rational, so $\mathrm{M}_{d}^{n}$ is unirational, which for some applications, such as the density of points defined over a number field $K$, is enough. However, in order to investigate the structure of $\mathrm{M}_{d}^{n}$ we need more than that. We do not expect a result along the lines of that in [13], that $\mathrm{M}_{2} \cong \mathbb{A}^{2}$, but we do expect rationality of $\mathrm{M}_{d}^{n}$. 
I would like to express my gratitude to my advisor Shouwu Zhang for introducing me to dynamical systems and guiding my research, to Lucien Szpiro and Joe Silverman for looking at the proofs of the major theorems in this paper, and to Xander Faber for helping me with this paper's presentation.

\section{The Spaces $\operatorname{Hom}_{d}^{n}$ and $\mathrm{M}_{d}^{n}$}

The space $\operatorname{Hom}_{d}^{n}$ of degree- $d$ morphisms on $\mathbb{P}^{n}$ arises as the subset of $\mathbb{P}^{N}=\left\{\left(q_{0}: q_{1}\right.\right.$ : $\left.\left.\ldots: q_{n}\right)\right\}$ defined by the condition that the $q_{i}$ 's share no common root. In order to give this space an algebraic structure, we investigate its complement. We will show the following result, proven by Macaulay [4] and reinterpreted here in modern language:

Theorem 2.1. The maps on $\mathbb{P}^{n}$ of degree $d$ such that the $q_{i}$ 's share a nonzero root form a closed, irreducible subvariety of $\mathbb{P}^{N}$ of codimension 1 , which is defined over $\mathbb{Z}$.

Proof. Consider the variety $V=\mathbb{P}^{n} \times \mathbb{P}^{N}$. We think of $V$ as representing a set of polynomials $\left(q_{0}: q_{1}: \ldots: q_{n}\right)$ acting on the point $\left(x_{0}: x_{1}: \ldots: x_{n}\right)$. Consider the resultant subvariety $U \subset V$ defined by the condition that $q_{i}(\mathbf{x})=0$ for all $i$. This variety clearly has codimension at most $n+1$. If we denote the variables defining $\mathbb{P}^{N}$ as $a_{j_{0}^{i} j_{1}^{i} \ldots j_{n}^{i}}^{i}$ with $j_{0}^{i}+\ldots+j_{n}^{i}=d$, representing the $x_{0}^{j_{0}^{i}} \ldots x_{n}^{j_{n}^{i}}$ monomial of $q_{i}$, then we see that $U$ is defined by equations that are bihomogeneous of degree 1 in the $a_{J}^{i}$ 's and $d$ in the $x_{i}$ 's.

We claim that $U$ is irreducible. The claim follows from a generalization of the fact that a primitive polynomial is irreducible over a domain whenever it is irreducible over its fraction field. More precisely, let $R$ be a domain with fraction field $K$, and let $I$ be an ideal of $R\left[y_{1}, \ldots, y_{m}\right]$ that is not contained in any prime of $R$. We have a natural map $f$ from $\operatorname{Spec} K\left[y_{1}, \ldots, y_{m}\right]$ to $\operatorname{Spec} R\left[y_{1}, \ldots, y_{m}\right]$. If $V(I)$ is reducible over $R$, say $V(I)=V_{1} \cup V_{2}$ with $V_{i}$ nonempty, then either $V(I)$ is reducible over $K$, or one $f^{-1}\left(V_{i}\right)$, say $f^{-1}\left(V_{1}\right)$, is empty. In the latter case, $I\left(V_{1}\right)$ may not contain nonconstant polynomials, so it contains at least one prime constant. This contradicts the assumption that $I$ is not contained in any prime of $R$; hence, $V(I)$ is reducible over $K$.

With the above generalization, suppose that $U$ is reducible. Then it is also reducible as a subvariety of $\mathbb{A}^{n+1} \times \mathbb{A}^{N+1}$. Further, by letting $R=\mathbb{Z}\left[x_{0}, \ldots, x_{n}\right]$ and $K$ be its fraction field, we see that either $U$ is contained in a prime of $R$, or $U$ is reducible in

$\mathbb{A}_{K}^{N+1}$. The former case is impossible since $U$ is not contained in any prime of $\mathbb{Z}$ or any relevant prime ideal of the ring of polynomials over $\mathbb{Z}$, and the latter is impossible since it is defined by linear equations in the $a_{J}^{i}$ 's. Either way this is a contradiction, so $U$ is irreducible and the claim is proven.

Finally, the maps on $\mathbb{P}^{n}$ of degree $d$ whose polynomials have a common nonzero root arise as the projection of $U$ onto the second factor of $\mathbb{P}^{n} \times \mathbb{P}^{N}$. It is irreducible because the projection map is surjective. It is closed because the map is proper. It has codimension at most 1 because almost all polynomials in $U$ share just one root, so 
that the dimension of $U$ and its image are equal. It has exact codimension 1 because some maps, for instance $q_{i}=x_{i}^{d}$, are morphisms. And it is defined over $\mathbb{Z}$ because every construction we have made in this proof is defined over $\mathbb{Z}$.

We call the image of $U$ the resultant subvariety of $\mathbb{P}^{N}$; we call its generating polynomial the Macaulay resultant and denote it by $\operatorname{Res}_{d}^{n}$. Macaulay proved the theorem by constructing the resultant explicitly, and showing that it has integer coefficients and is irreducible. His explicit construction shows that if the polynomials are homogeneous of degrees $d_{0}, d_{1}, \ldots, d_{n}$, then the resultant is $(n+1)$-homogeneous in the coefficients of each polynomial $p_{i}$ of degree $\prod_{j \neq i} d_{j}$. In our case, all the degrees are equal to $d$, so that the resultant is $(n+1)$-homogeneous in the coefficients of each $q_{i}$ of degree $d^{n}$. In particular, the resultant subvariety is a hypersurface of degree $(n+1) d^{n}$.

Theorem 2.1 shows that the space of morphisms is the complement of the resultant subvariety, and is therefore affine and of dimension $N$. Silverman [13], who only considers the case $n=1$, refers to this space as Rat $d$; we will refer to it as $\operatorname{Hom}_{d}^{n}$ and to its complement in $\mathbb{P}^{N}$ as $\operatorname{Res}_{d}^{n}$ by abuse of notation.

The action of $\operatorname{PGL}(n+1)$ on $\mathbb{P}^{n}$ leads to a conjugation action on $\operatorname{Hom}_{d}^{n}$, wherein $A \in \operatorname{PGL}(n+1)$ acts on a rational map $\varphi$ by sending it to $A \varphi A^{-1}$. The property of being ill-defined at a point is stable under both the left action mapping $\varphi$ to $A \varphi$ and the right action mapping $\varphi$ to $\varphi A^{-1}$; hence, the conjugation action is well-defined on $\operatorname{Hom}_{d}^{n}$. The space of endomorphisms of $\mathbb{P}^{n}$ defined by degree- $d$ polynomials may be regarded as the quotient of $\operatorname{Hom}_{d}^{n}$ by the conjugation action.

A priori, we only know that over an algebraically closed field, the quotient exists as a set. In order to give it algebraic structure, we need to pass to the stable or semistable space in geometric invariant theory [8]. Fortunately, we have the following result:

Theorem 2.2. Every $\varphi \in \operatorname{Hom}_{d}^{n}$ is stable.

Proof. We use the Hilbert-Mumford criterion, as described in chapter 2 of [8]. To do that, we pull back the action of $\operatorname{PGL}(n+1)$ on $\mathbb{P}^{N}$ to the action of $\operatorname{SL}(n+1)$ on $\mathbb{A}^{N+1}$, and consider one-parameter subgroups of $\operatorname{SL}(n+1)$. The criterion states that a point lies in the stable space $\operatorname{Hom}_{d}^{n, s}$ (respectively, the semistable space $\operatorname{Hom}_{d}^{n, s s}$ ) iff for every such subgroup, its action on the point can be diagonalized with diagonal elements $t^{a_{I}}$, and at least one $a_{I}$ is negative (resp. non-positive).

Note that the action of $A \in \operatorname{SL}(n+1)$ on $\varphi \in \mathbb{A}^{N+1}$ is conjugate to the action of $B A B^{-1}$ on $B \varphi B^{-1}$. In particular, it will have the same eigenvalues, so the action of a one-parameter subgroup $G=\mathbb{G}_{m}$ will have the same $a_{I}$ 's. Therefore, we may conjugate $G$ to be diagonal, which will be enough to give us criteria for stability and semistability up to conjugation. So from now on, we assume $G$ is the diagonal subgroup whose $i$ th diagonal entry is $t^{a_{i}}, a_{i} \in \mathbb{Z}$. Here we label the rows and columns from 0 to $n$, in parallel with the label for the $q_{i}$ 's. We have $a_{0}+\ldots+a_{n}=0$. We may also assume that $a_{0} \geq a_{1} \geq \ldots \geq a_{n}$, after conjugation if necessary, and that the $a_{i}$ 's are coprime. 
The action of $G$ on $\mathbb{A}^{N+1}$ is already diagonal. We denote the $\mathbf{x}^{\mathbf{d}}$ coefficient of $q_{i}$ by $c_{\mathbf{d}}(i)$; then $G$ multiplies $c_{\mathbf{d}}(i)$ by $t^{a_{i}} t^{-\left(a_{0} d_{0}+\ldots+a_{n} d_{n}\right)}$. A point $\varphi$ is not stable (resp. unstable) if for some choice of $G$, all the $c_{\mathbf{d}}(i)$ 's for which $a_{0} d_{0}+\ldots+a_{n} d_{n}>a_{i}$ (resp. $\left.a_{0} d_{0}+\ldots+a_{n} d_{n} \geq a_{i}\right)$ are zero. Let us observe that this means that, for $d>1$, every $x_{0}^{d}$ coefficient has to be zero, as we will have $d a_{0}>a_{0} \geq a_{i}$ for every $i$. This means that $\varphi$ lacks any $x_{0}^{d}$ coefficient, so that the $q_{i}$ 's have a nontrivial zero at $(1: 0: \ldots: 0)$, and $\varphi \notin \operatorname{Hom}_{d}^{n}$. The property of not being a morphism is preserved under conjugation, proving the theorem.

Since $\operatorname{Hom}_{d}^{n}$ is stable, it has a natural geometric quotient induced by the $\operatorname{PGL}(n+1)$ action on $\mathbb{P}^{N}$, which we denote by $\mathrm{M}_{d}^{n}$; as $\operatorname{Hom}_{d}^{n}$ is affine, $\mathrm{M}_{d}^{n}$ is affine, with structure sheaf $\mathcal{O}_{\mathrm{Hom}_{d}^{n}}^{\mathrm{SL}(n+1)}$. We may also write $\mathrm{M}_{d}^{n, s}$ for the quotient of the stable space and $\mathrm{M}_{d}^{n, s s}$ for the quotient of the semistable space. The latter quotient is only categorical, rather than geometric, but will be proper over $\operatorname{Spec} \mathbb{Z}$ (all spaces in question, as well as $\mathrm{SL}(n+1)$, are defined over $\mathbb{Z}$; hence, so are the quotients).

Let us now describe the not-stable and unstable spaces more explicitly. In the $n=1$ case, $G$ depends only on $a_{0}$, which may be taken to be 1 . This gives us only one criterion for stability (resp. semi-stability), which means that the not-stable (resp. unstable) space is irreducible (in fact, it will be a linear subvariety and its orbit under PGL(2)-conjugation). When $n>1$, this is no longer true: $G$ depends on multiple variables, and we can find many infinite families of coprime $a_{i}$ 's that sum to 0 and are in decreasing order.

However, the not-stable (resp. unstable) space will still be a union of finitely many linear subvarieties and their PGL $(n+1)$ conjugates, whose number will generally grow with $d$ and $n$. This is because there are only $2^{N+1}$ linear spaces defined by conditions of the form $c_{\mathbf{d}}(i)=0$ for a collection $J$ of $(\mathbf{d}, i)$ pairs. For each such space, either there exists a $G$ such that $(\mathbf{d}, i) \in J$ if and only if $a_{0} d_{0}+\ldots+a_{n} d_{n}>a_{i}$ (resp. $a_{0} d_{0}+\ldots+a_{n} d_{n} \geq a_{i}$ ), or there doesn't. Of course, a given $J$ may correspond to infinitely many $G$, which will in general have ratios $a_{0}: \ldots: a_{n}$ that are close in the archimedean metric.

We omit the calculation of the linear subvarieties that occur as the not-stable (resp. unstable) space for each $d$ and $n$, as well as the number of such varieties. We will just note that there are far fewer than $2^{N+1}$ such varieties: for a start, we have already seen that $((d, 0, \ldots, 0), i) \in J$ for all $i$. One more constraint that follows trivially from the definition of the $a_{i}$ 's is that if $(\mathbf{d}, i) \in J$, then so is $(\mathbf{d}, j)$ for $j>i$. Put another way, not being stable (resp. instability) imposes more conditions on $q_{j}$ than on $q_{i}$ for $j>i$. It may also be shown that for each $G$ the number of conditions is roughly between one half and $e^{-1}$ times $N$; we omit the proof, as this result will not be relevant in the remainder of this paper.

Finally, when $n=1$, the only $G$ has $a_{0}=1, a_{1}=-1$, so $a_{0} d_{0}+a_{1} d_{1}=d_{0}-d_{1}=$ $2 d_{0}-d$. When $d$ is even, $2 d_{0}-d$ is always even, so the conditions $a_{0} d_{0}+a_{1} d_{1}>a_{i}$ and $a_{0} d_{0}+a_{1} d_{1} \geq a_{i}$ coincide, and the stable and semistable spaces are the same; this was shown in [13]. We will show that this will never be the case for higher $n$. 
First, observe that if we set $a_{0}=1, a_{n}=-1$, and $a_{i}=0$ for $i \neq 0, n$, we obtain $a_{0} d_{0}+\ldots+a_{n} d_{n}=d_{0}-d_{n}$, which may take any value between $-d$ and $d$ inclusive. Hence, the conditions $a_{0} d_{0}+\ldots+a_{n} d_{n}>a_{i}$ and $a_{0} d_{0}+\ldots+a_{n} d_{n} \geq a_{i}$ will not coincide.

Now, suppose that $\varphi$ is a point that is not stable, with $c_{\mathbf{d}}(i)=0$ if and only if $d_{0}-d_{n}>a_{i}$ with $a_{i}$ as above. If $\varphi$ is unstable, then we can find some $G$ such that if $a_{0} d_{0}+\ldots+a_{n} d_{n} \geq a_{0}$ then $d_{0}-d_{n}>1$, and if $a_{0} d_{0}+\ldots+a_{n} d_{n} \geq a_{i}$ for $i \neq 0, n$, then $d_{0}-d_{n}>0$. If for that $G$ we have $a_{1} \geq 0$, then looking at the $x_{0} x_{1}^{\bar{d}-1}$ monomial, we get $a_{0} d_{0}+\ldots+a_{n} d_{n}=a_{0}+(d-1) a_{1} \geq a_{0}$ but $d_{0}-d_{n}=1$, a contradiction. If $a_{1}<0$, then we must have $a_{i}<0$ for all $i>0$, so $a_{0}+a_{n}>0$. For $d=2 k+1$, we consider the $x_{0}^{k+1} x_{n}^{k}$ monomial, for which $a_{0} d_{0}+\ldots+a_{n} d_{n}=k\left(a_{0}+a_{n}\right)+a_{0}>a_{0}$ but $d_{0}-d_{n}=1$; for $d=2 k$, we consider the $x_{0}^{k} x_{n}^{k}$ monomial, for which $a_{0} d_{0}+\ldots+a_{n} d_{n}=k\left(a_{0}+a_{n}\right)>0>a_{1}$ but $d_{0}-d_{n}=0$. Either way, we have a contradiction, so $\varphi$ is semistable but not stable. This proves:

Proposition 2.3. For all $d, n>1$, we have $\operatorname{Hom}_{d}^{n, s} \subsetneq \operatorname{Hom}_{d}^{n, s s}$.

We will conclude this section with the following strict containment:

Proposition 2.4. $\operatorname{Hom}_{d}^{n} \subsetneq \operatorname{Hom}_{d}^{n, s}$.

Proof. Observe that the linear subvarieties defined above are invariant under conjugation by every upper triangular matrix, at least when we ensure $a_{0} \geq a_{1} \geq \ldots \geq a_{n}$. Hence, the codimension of the not-stable space is equal to the codimension of the largest linear subvariety, minus $\frac{n(n+1)}{2}$. It suffices to show this codimension is more than 1 , or, in other words, that every linear subvariety has codimension at least $\frac{n(n+1)}{2}+2$. We will consider two cases.

Case 1. $a_{1} \geq 0$. When $d_{0}>0$, the $x_{0}^{d_{0}} x_{1}^{d_{1}}$ monomial has $a_{0} d_{0}+a_{1} d_{1}>a_{1}$, so it is zero for all $q_{i}$ 's except $q_{0}$; when $d_{0}>1$ it is also zero for $q_{0}$, since $a_{0} d_{0}+a_{1} d_{1} \geq 2 a_{0}$. This gives us a total codimension of $n^{2}+(n-1)$, which is larger than $\frac{n(n+1)}{2}+1$ for all $n \geq 2$. When $n=1$ this case is impossible because we need to have $a_{0}+a_{1}=0$.

Case 2. $a_{1}<0$. We have $a_{0}=-\left(a_{1}+\ldots+a_{n}\right)>-a_{i}$ for all $i$; therefore, the $x_{0}^{d-1} x_{i}$ monomial is zero in every $q_{j}$ except $q_{0}$; the $x_{0}^{d}$ monomial is always zero. This gives us a codimension of $n^{2}+n+1$, which is large enough for all $n$.

Remark 2.5. The larger spaces $\operatorname{Hom}_{d}^{n, s}$ and $\operatorname{Hom}_{d}^{n, s s}$ have a meaning in the field of moduli spaces more than in this of dynamical systems, where we study the iterates of morphisms. The problem is that we cannot always iterate rational maps which are not morphisms, even if they are stable: the image may not be dense, and may eventually map to a locus on which the map is ill-defined. A map of the form $(q: 0: 0: \ldots: 0)$ with $q(1,0, \ldots, 0)=0$ will be impossible to iterate. For general $q$, it will also be stable for large $d$, because we will have $a_{0} d_{0}+\ldots+a_{n} d_{n}>a_{0}$ for many different $\mathbf{d}$ 's no matter how we choose the $a_{i}$ 's, even after conjugation. When $n=1$, it suffices to have $d \geq 4$, because then $\varphi$ is unstable only if is of the form $(p: q)$ with $p$ and $q$ sharing a common root of multiplicity at least $\frac{d-1}{2}$, and we may pick a map $(q: 0)$ with $q$ having distinct roots. For one approach for giving a completion of $\operatorname{Hom}_{d}^{n}$ in a way that permits iteration at the boundary, see [2]. 


\section{Stabilizer Groups}

The moduli space $\mathrm{M}_{d}^{n}$, as well as its stable and semistable completions, has a welldefined function mapping each morphism to its stabilizer group in $\operatorname{PGL}(n+1)$, which will be well-defined up to conjugation. This stabilizer will be finite, at least on $\mathrm{M}_{d}^{n, s}$, from standard facts from geometric invariant theory. We will study the possible subgroups of PGL $(n+1)$ that may occur as stabilizers of morphisms. We gain very little by assuming Theorem 2.2, so we might as well not assume it a priori; this will provide an alternative proof for it.

Note that the resultant is a $\mathrm{PGL}(n+1)$-invariant section of a $\mathrm{PGL}(n+1)$-linearizable divisor on $\mathbb{P}^{N}$ that is nonzero on $\operatorname{Hom}_{d}^{n}$. Therefore, on $\operatorname{Hom}_{d}^{n}$ stability is equivalent to having closed fibers, which is equivalent to having a stabilizer group of the lowest possible dimension (see chapter 1 of [8]). Hence, to provide a second proof of Theorem 2.2, it suffices to show that the stabilizer of every $\varphi \in \operatorname{Hom}_{d}^{n}$ is finite. This was done in [10]. We will prove a stronger result:

Theorem 3.1. The stabilizer of every point in $\operatorname{Hom}_{d}^{n}, d>1$, is a finite group of order bounded in terms of $n$ and $d$.

Proof. Note that if $A \in \operatorname{Stab}(\varphi)$, then $B A B^{-1} \in \operatorname{Stab}\left(B \varphi B^{-1}\right)$. Therefore, when considering individual stabilizing matrices, we may assume they are in Jordan canonical form. We use the following result:

Lemma 3.2. If $A \in \operatorname{Stab}(\varphi)$, and $\varphi$ is not purely inseparable, then $A$ is diagonalizable.

Proof. In characteristic zero, this is trivial given Theorem 2.2. However, it is not trivial in characteristic $p$; the proof works for every characteristic, so we lose nothing from not using Theorem 2.2.

We will assume that $A$ is not diagonalizable and derive a contradiction. It suffices to assume that $A$ is a Jordan matrix whose largest Jordan block is of size $r>1$. After conjugation and scaling, we may assume that the first Jordan block is also the largest, and has eigenvalue 1 . We will label the rows and columns from 0 to $n$, in parallel with the labels for the $q_{i}$ 's. We will also write $\varphi=\left(q_{0}: q_{1}: \ldots: q_{n}\right), k_{i}=a_{i i}$ for the eigenvalue in the $i$ th position, and $r_{i}$ for the size of the Jordan block containing $a_{i i}$. We have $r_{0}=r, k_{0}=1, r_{i} \leq r$.

Note that the inverse of the first Jordan block is the matrix with zeroes below the main diagonal and $a_{i j}=(-1)^{i-j}$ on or above it. Therefore, each vector $\mathbf{x}=$ $\left(x_{0}, x_{1}, \ldots, x_{n}\right)$ is transformed to:

$$
\mathbf{x}^{\prime}=\left(x_{0}-x_{1}+\ldots \pm x_{r-1}, x_{1}-x_{2}+\ldots \mp x_{r-1}, \ldots, x_{r-1}, \ldots, \frac{1}{k_{n}} x_{n}\right)
$$

We write $q_{i}^{\prime}(\mathbf{x})=q_{i}\left(\mathbf{x}^{\prime}\right)$. Similarly, $A$ transforms $\varphi=\left(q_{0}, \ldots, q_{n}\right)$ to:

$$
\varphi^{\prime}=\left(q_{0}^{\prime}+q_{1}^{\prime}, q_{1}^{\prime}+q_{2}^{\prime}, \ldots, q_{r-1}^{\prime}, \ldots, k_{n} q_{n}^{\prime}\right)
$$

Since $A$ stabilizes $\varphi$, we need $\varphi^{\prime}$ to be a scalar multiple of $\varphi$. 
For each $\mathbf{d} \in \mathbb{Z}^{n+1}$, we denote the $\mathbf{x}^{\mathbf{d}}$ coefficient of $q_{i}$ (respectively $q_{i}^{\prime}$ ) by $c_{\mathbf{d}}(i)$ (resp. $c_{\mathbf{d}}^{\prime}(i)$ ). We suppress trailing zeroes for simplicity, so that $c_{d}$ denotes the $x_{0}^{d}$ coefficient. We are looking for the largest $i$ such that $c_{d}(i) \neq 0$; such an $i$ exists, or else $(1: 0: \ldots: 0)$ is a common root of all the $q_{i}$ 's. As the only $x_{0}^{d}$ term in $\mathbf{x}^{\prime \mathbf{d}}$ comes from $x_{0}^{\prime d}$, we have $c_{d}^{\prime}(j)=c_{d}(j)$ for all $j$. Now in $\varphi^{\prime}$, the $i$ th term is either $q_{i}^{\prime}$ or $q_{i}^{\prime}+k_{i} q_{i+1}^{\prime}$, so that its $x_{0}^{d}$ coefficient is $k_{i} c_{d}(i)$. This implies that the scaling factor is $k_{i}$, i.e. $\varphi^{\prime}=k_{i} \varphi$.

Now, assume that $i$ is not at the beginning of its Jordan block, that is that $a_{i-1, i}=1$. Then $k_{i-1}=k_{i}$, and the fact that $\varphi^{\prime}=k_{i} \varphi$ implies that $k_{i-1} c_{d}^{\prime}(i-1)+c_{d}^{\prime}(i)=k_{i} c_{d}(i-1)$. This reduces to $c_{d}(i)=0$, a contradiction. Therefore, $i$ is at the beginning of its Jordan block.

Let us now consider the $x_{0}^{d-1} x_{1}$ coefficients, and assume throughout that all indices are in the same Jordan block as $i$. We have $c_{d-1,1}^{\prime}(j)=c_{d-1,1}(j)-d c_{d}(j)$. For $j>i$, this reduces to $c_{d-1,1}^{\prime}(j)=c_{d-1,1}(j)$. Conversely, the corresponding term to $c_{d-1,1}$ in $\varphi^{\prime}=k_{i} \varphi$ will be $k_{i} c_{d-1,1}^{\prime}(j)+c_{d-1,1}^{\prime}(j+1)=k_{i} c_{d-1,1}(j)$. When $j>i$, this implies that $c_{d-1,1}^{\prime}(j+1)=0$, so that $c_{d-1,1}(j)=0$ for $j>i+1$; conversely, for $i+1$, we obtain $k_{i} c_{d-1,1}^{\prime}(i)+c_{d-1,1}^{\prime}(i+1)=k_{i} c_{d-1,1}(i)$, which reduces to $c_{d-1,1}(i+1)=k_{i} d c_{d}(i) \neq 0$. This shows that $i+1$ is the largest index with a nonzero $x_{0}^{d-1} x_{1}$ coefficient, at least in the Jordan block containing $i$.

We may apply induction on $s(\mathbf{d})=d_{1}+2 d_{2}+\ldots+(r-1) d_{r-1}$, and find that in the Jordan block containing $i$, the largest index with a nonzero $\mathbf{x}^{\mathbf{d}}$ coefficient is $i+s(\mathbf{d})$. Note that the Jordan block has $r_{i} \leq r$ elements, but the number of monomial indices attached to the first Jordan block is $(r-1) d+1$, which is strictly greater than $r$ when $d, r>1$. This is a contradiction: the last element of the Jordan block has $k_{i} c_{\mathbf{d}}^{\prime}=k_{i} c_{\mathbf{d}}$ for all d d.e. $c_{\mathbf{d}}\left(i+r_{i}-1\right)^{\prime}=c_{\mathbf{d}}\left(i+r_{i}-1\right)$, but that last equality is only true when $s(\mathbf{d}) \leq r_{i}$, which is not the case for all $\mathbf{d}$. Since we are assuming $d>1$, we must have $r=1$, and we are done.

The careful reader may note that the proof that $i+s$ is the largest index with a nonzero $\mathbf{x}^{\mathbf{d}}$ coefficient for $s(\mathbf{d})=s$ makes an assumption about the characteristic we are working in. In characteristic zero, $d \neq 0$ and there is no problem. In characteristic $p$, we need to treat separately the case when $p<d$. Then for example we may have $p \mid d$, so that $c_{d-1,1}^{\prime}(j)=c_{d-1,1}(j)$ for all $j$, and $c_{d-1,1}(i+1)$ may be zero. Note that the number of monomial indices containing $x_{0}^{d-2}$ attached to the first Jordan block is $2(r-1)+1$, which is strictly greater than $r$ when $r>1$; when $p \nmid d(d-1)$, we may restrict ourselves to such monomials, and the proof proceeds as in characteristic zero.

When $p \mid d-1$, we may restrict ourselves to monomials containing $x_{0}^{d-1}$, and proceed with the proof. We will only encounter an obstruction if $r_{i}=r$ and only at the end of the Jordan block, where the existence of a nonzero $x_{0}^{d-1} x_{r-1}$ monomial does not guarantee that of $x_{0}^{d-2} x_{1} x_{r-1}$. However, the action of $A$ on $q_{i+r-1}$ takes it to $k_{i} q_{i+r-1}^{\prime}$, and we must have $c_{\mathbf{d}}^{\prime}(i+r-1)=c_{\mathbf{d}}(i+r-1)$ for all $\mathbf{d}$. If we write $d-1=p^{l} m, m \nmid p$, then we see that $x_{0}^{d-1} x_{r-1}$ is transformed to $k_{i}\left(x_{0}-x_{1}+\ldots \pm\right.$ $\left.x_{r-1}\right)^{d-1} x_{r-1}=k_{i}\left(x_{0}^{p^{l}}-\ldots \pm x_{r-1}^{p^{l}}\right)^{m} x_{r-1}$ which shows that the $x_{0}^{p^{l}(m-1)} x_{1}^{p^{l}}$ monomial does not satisfy $c_{\mathbf{d}}^{\prime}(i+r-1)=c_{\mathbf{d}}(i+r-1)$. This yields a contradiction. 
Finally, when $p \mid d$, we may write $d=p^{l} m$. When $m>1$, we apply exactly the same proof as in characteristic zero, except that we write $m$ instead of $d$ and $m_{j}=\frac{d_{j}}{p^{l}}$ instead of $d_{j}$; then we define $s(\mathbf{d})=m_{1}+\ldots+(r-1) m_{r-1}$, and in the Jordan block containing $i$, the largest index with a nonzero $\mathbf{x}^{\mathbf{d}}$ coefficient is $i+s(\mathbf{d})$. As $m>1$, we have $(r-1) m+1>r$ for $r>1$, and we have the same contradiction as in the characteristic zero case. Note that when $m=1$, we may derive the same contradiction from any nonzero monomial not of the form $x_{j}^{d}$, which must exist if $\varphi$ is not purely inseparable. Hence, if $\varphi$ has a non-diagonalizable stabilizer then it is purely inseparable and we are done.

With the above lemma, we know that any abelian subgroup of $\operatorname{Stab}(\varphi) \in \operatorname{GL}(n+1)$ will be simultaneously diagonalizable. We will prove the following uniform bound on the size of abelian stablizing subgroups:

Lemma 3.3. Every diagonal subgroup stabilizing $\varphi \in \operatorname{Hom}_{d}^{n}$ is of size at most $d^{n+1}$.

Proof. A diagonal matrix $A$ with diagonal entries $\left(a_{0}, a_{1}, \ldots, a_{n}\right)$ acts on each $q_{i}$ by multiplying $c_{\mathbf{d}}(i)$ by $\frac{a_{i}}{a_{0}^{d_{0}} \ldots a_{n}^{d_{n}}}$. Our case of interest will be the $x_{j}^{d}$ coefficients. Each has to be nonzero for at least one $i$, which induces the equation $a_{i}=a_{j}^{d}$. Note that we may set the scaling factor $k$ to be 1 , since the scalar matrix $k^{\frac{1}{1-d}}$ multiplies every coefficient by $k$.

Now, we have at least $n+1$ different relations $a_{i}=a_{j}^{d}$. We may drop relations until each $j$ has just one $i$ such that such a relation holds; dropping relations will increase the size of the group, so by bounding the size of the larger group, we will bound the size of any automorphism group.

We obtain a function $j \mapsto i$. If the function is bijective, we may write it as a product of disjoint cycles, and conjugate to get the cycles to be $\left(\begin{array}{llll}0 & 1 & \ldots & s_{1}-1\end{array}\right) \ldots\left(n-s_{k}+\right.$ $1 \ldots n$ ), where here $r_{i}$ denotes the length of the $i$ th cycle, and has nothing to do with the definition in Lemma 3.2. Then $a_{0}^{d^{r} 1}=a_{0}$ and $a_{0}$ is a root of unity of order dividing $d^{r_{1}}-1$, the choice of which uniquely determines $a_{i}, 0 \leq i \leq r_{1}-1$. We have similar results for $a_{r_{1}}, \ldots, a_{n-r_{k}+1}$; since $\sum r_{i}=n+1$, this bounds the size of the group by $d^{n+1}$.

In general, of course, the function $j \mapsto i$ may not be bijective, so we can only write it as a product of precycles, whose cycles are disjoint. Here a precycle means a cycle and zero or more tails. The above discussion applies to the cycles. For the tails, suppose without loss of generality that $\left(\begin{array}{llll}0 & 1 & \ldots\end{array}\right)$ is a tail where $r$ and no element before it is part of a cycle; then the choice of $a_{r}$ determines a choice of $d$ possibilities for $a_{r-1}$ and in general $d^{s}$ for $a_{r-s}$ subject to the obvious compatibility condition. This clearly respects the bound of $d^{n+1}$ : if $m$ is the total number of elements in cycles, then we have at most $m^{n+1}$ possibilities for the cycles, each of which gives us exactly $(d-m)^{n+1}$ possibilities for the tails.

The bound $d^{n+1}$ works for abelian stabilizing subgroups in the purely inseparable case as well. We may view a purely inseparable $\varphi$ as the action of raising every 
coefficient to the $d$ th power followed by the matrix $B$. Then $A \varphi A^{-1}=\varphi$ if and only if $A B A_{d}^{-1}=B$, where $A_{d}$ is the image of the matrix $A$ under the homomorphism of raising every entry to the $d$ th power; we need to show the group of such $A$, which we will write as $\operatorname{Stab}(B)$, is finite. Since $A$ and $A_{d}$ are conjugate, all eigenvalues of $A$ are in $\mathbb{F}_{d}$.

We may conjugate an abelian stabilizing subgroup $G$ to obtain a block diagonal group with each block upper triangular and with its $(i, j)$ entry depending only on $j-i$. We may also fix one element, $C$ to be in Jordan canonical form, in which case we will have $C_{d}=C$ and thus $B C=C B$. Then $B$ is in block form; labeling the blocks by $r, s$ and the $r$ th block of $C$ by $C_{r}$, we see that the $B_{r s}$ is nonzero if and only if the blocks $r$ and $s$ are of the same size and equal for every element of $\operatorname{Stab}(B)$, and in any case $B_{r s}$ commutes with $C_{r}=C_{s}$, so it is upper triangular with its $(i, j)$ entry depending only on $j-i$. In particular, it commutes with every $A_{r}=A_{s}$, so that $B$ commutes with $G$. Hence for all $A \in G$, we have $A B=B A$ and $A B A_{d}^{-1}=B$, so that $A=A^{d}$ and $A$ has entries in $\mathbb{F}_{d}$. Furthermore, for each block in $G$ of size $r$, we have $r$ positive possibilities for $j-i$, inducing $d^{r}$ possible blocks, and $d^{n+1}$ possible matrices in $G$.

Note that we may have additional stabilizing matrices in $\operatorname{PGL}(n+1)$. These occur when there exists an automorphism of the set $\{0,1, \ldots, n\}$ that does not leave the diagonal vector $\mathbf{a}=\left(a_{0}, \ldots, a_{n}\right) \in \mathbb{A}^{n+1}$ fixed, but does fix $\mathbf{a}=\left(a_{0}: \ldots: a_{n}\right) \in \mathbb{P}^{n}$. Since the automorphism has to fix $a_{0} a_{1} \ldots a_{n}$, we see that it must send each $a_{i}$ to $\zeta a_{i}$ where $\zeta$ is a root of unity of order at most $n+1$; hence there are at most $n+1$ possibilities for such an automorphism, modulo automorphisms that fix $\mathbf{a} \in \mathbb{A}^{n+1}$ and are hence simultaneously block-diagonalizable with $A$.

We will rely on one final bound, due to G. A. Miller [6]:

Proposition 3.4. The size of a finite group is bounded in terms of the size of its largest abelian subgroup.

Proof. It suffices to show this for $p$-groups. For each $n$, we let $k(n)$ be the minimal exponent of the largest abelian subgroup of any $p$-group of exponent $n$. Furthermore, for each $l \leq n$, we let $k(n, l)$ be the minimal exponent subject to the restriction that $Z=Z(G)$ have exponent $l$, so that $k(n)=\min \{k(n, l)\}$. It is enough to show that $\lim _{n \rightarrow \infty} k(n)=\infty$.

It is trivial to show that $k(2)=2$. In general, for a $p$-group of exponent $n$ and center of exponent $l$, let $g$ be such that $g \notin Z, g^{p} \in Z$, and $g Z \in Z(G / Z)$. Unless $G$ is abelian, in which case the result is trivial, we may take $g$ to be a preimage of a nontrivial element in the socle of $G / Z$. For every $h \in G, h g h^{-1}=g z$ for some $z \in Z$; we obtain a group homomorphism $h \mapsto z$ from $G$ to $Z$. The homomorphism has kernel $K$ of exponent at least $n-l$ and center containing $\langle Z, g\rangle$. Any abelian subgroup of $K$ will be an abelian subgroup of $G$, so that we obtain $k(n, l) \geq k(n-l, l+1)$. It easily follows that $k(n) \geq 2 \sqrt{n}$.

The bound in the above proposition is very weak. It is known that for odd $p$ we have $k(n) \leq \frac{n+4}{3}$ and for $p=2$ we have $k(n) \leq 2 \frac{n+3}{5}[1$, but little more. It is also 
not known a priori that the group has to be finite, only that if it is finite then it is bounded. We may use Theorem 2.2 and finish. However, with little additional effort, we may prove finiteness directly, providing an alternative proof that all morphisms are stable. The fact that finite implies uniformly bounded means that it is enough to show that every finitely generated stabilizing subgroup is finite. More precisely:

Proposition 3.5. Every finitely generated subgroup of $\mathrm{PGL}(n)$ contained in finitely many finite-order conjugacy classes is finite.

Proof. Let $R$ be the $\mathbb{Z}$-algebra generated by the finitely many coefficients of the generators. Then the group is contained in $\operatorname{PGL}(n, R)$, and we may project it into the finite group $\operatorname{PGL}(n, R / \mathfrak{m})$ where $\mathfrak{m}$ is a maximal ideal in $R$; we will show the map can be chosen to be injective. In fact, each non-unipotent conjugacy class $i$ contains two different eigenvalues, $a_{i_{1}}, a_{i_{2}}$; therefore, if we choose $\mathfrak{m}$ not to contain $a_{i_{1}}-a_{i_{2}}$, which we can since there are only finitely many such elements, then the map will have unipotent kernel. In characteristic 0 , the only finite-order unipotent matrix is the identity, so the map is injective and we are done.

In characteristic $p$, we obtain a finite-index and hence finitely generated unipotent group. We may conjugate it by some matrix $P$ to be upper triangular; then matrix multiplication is equivalent to addition of the $(r, r+1)$ entry for any $r$, and the finite generation implies that the set of all $(r, r+1)$ entries lies in a finitely generated $\mathbb{Z} / p \mathbb{Z}$ vector space, which is finite. For the matrices with all $(r, r+k)$ entries for all $k \leq l$, matrix multiplication corresponds to addition of $(r, r+l+1)$ entries, and we may add those entries to our vector space, which will remain finite. We may now construct $\mathfrak{m}$ to avoid the finite vector space and the determinant of $P$, as well as the eigenvalue differences described above. The map will then be injective.

Note that in the proof of proposition we make no assumption on the base ring. Of course, the argument in the proposition applies to $\mathrm{GL}(n+1)$, and shows that the answer to Burnside's problem, which asks whether a finitely generated group of bounded exponent is necessarily finite, is yes when restricted to subgroups with faithful finite-dimensional representations over any field.

For each stabilizer group $G \in \operatorname{PGL}(n+1)$, there is a closed subscheme $\operatorname{Fix}(G) \in$ $\operatorname{Hom}_{d}^{n}$ consisting of all $\varphi$ with stabilizer group containing $G$. Theorem 3.1 states that every $G$ with nonempty $\operatorname{Fix}(G)$ is finite and of bounded order. Furthermore, each nontrivial stabilizing matrix is, up to conjugation, one of the $d^{n+1}$ possibilities for each of the $(n+1)^{n+1}$ functions on the set $\{0,1, \ldots, n\}$. We may strengthen this result as follows:

Corollary 3.6. There are only finitely many $G$ with nonempty $\operatorname{Fix}(G)$ up to conjugation. In particular, on an open dense set of $\operatorname{Hom}_{d}^{n}$, which descends to $\mathrm{M}_{d}^{n}$, the stabilizer group is trivial.

Remark 3.7. The statement that there are only finitely many such $G$ up to conjugation is stronger than the statement that there are only finitely many $G$ up to isomorphism, which follows trivially from the bound on the size of $G$. 
Proof. Since the size of $G$ is bounded, it suffices to show that each stabilizing subgroup has finitely many projective $n+1$-dimensional representations up to conjugacy. This is always true when the representation is completely reducible, which will be true if the ambient characteristic $p$ does not divide $|G|$. But when $\operatorname{Fix}(G)$ is not purely inseparable, every element will be diagonalizable, so it will have order not divisible by $p$, so that $G$ has order not divisible by $p$. In the purely inseparable case, we have $\operatorname{PGL}(n+1)$ acting on itself stably and with finite stabilizers, so that each orbit is of dimension $(n+1)^{2}-1$ and thus consists of all of PGL $(n+1)$. In other words, every purely inseparable map is, up to conjugation, $\left(x_{0}^{d}: \ldots: x_{n}^{d}\right)$, so that its stabilizer group is conjugate to $\operatorname{PGL}\left(n+1, \mathbb{F}_{d}\right)$.

It remains to be shown that the complement of $\bigcup_{G \supset I} \operatorname{Fix}(G)$ is dense; its openness follows from the fact that the condition $A \varphi A^{-1}=\varphi$ is closed. It suffices to show that each $\operatorname{Fix}(G)$ is a proper subset of $\operatorname{Hom}_{d}^{n}$. We lose nothing if we ignore purely separable maps. From the proof of Lemma 3.3. each of the finitely many elements that may occur in $G$, a diagonal matrix with $i$ th entry $a_{i}$, multiplies $c_{\mathbf{d}}(i)$ by $\frac{a_{i}}{\mathbf{a}^{\mathrm{d}}}$, and hence induces the relation $c_{\mathbf{d}}(i)=0$ outside a set of $(\mathbf{d}, i)$ 's for which $\frac{a_{i}}{\mathbf{a}^{\mathrm{d}}}$ is constant. If $\frac{a_{i}}{\mathbf{a}^{\mathrm{d}}}$ is constant for all $(\mathbf{d}, i)$, then we have $a_{i}=k \mathbf{a}^{\mathbf{d}}$; choosing a constant $\mathbf{d}$, we see that $a_{i}$ is constant, so $A$ is a scalar matrix. Hence no non-trivial $A$ fixes all of $\operatorname{Hom}_{d}^{n}$.

Note that when $n=1$, [13] has an explicit bound on the size of $\operatorname{Stab}(\varphi)$ of $n_{1} ! n_{2} ! n_{3} !$, where the $n_{i}$ 's are indices for which there exist periodic points for $\varphi$ of exact order $n_{i}$. The technique in this paper improves on that bound. Following the proof of Lemma 3.3 , we have three possibilities for the map $j \mapsto i$ up to conjugation: $(1,2) \mapsto(1,2)$, $(1,2) \mapsto(2,1)$, and $(1,2) \mapsto(1,1)$. In the first case, $a_{0}=\zeta_{d-1}^{i}$ and $a_{1}=\zeta_{d-1}^{j}$, where we use $\zeta_{i}$ to denote an $i$ th root of unity; modulo multiplying both $a_{0}$ and $a_{1}$ by some $\zeta_{d-1}$, we obtain a cyclic group of order $d-1$. In the second case, we have $a_{0}=\zeta_{d^{2}-1}^{i}, a_{1}=a_{0}^{d}$, and modulo multiplying both by $\zeta_{d^{2}-1}^{d+1}$, we obtain a cyclic group of order $d+1$. In the third case, $a_{0}=\zeta_{d-1}$ and $a_{1}^{d}=a_{0}$, and modulo multiplying both by $\zeta_{d-1}$, we obtain a cyclic group of order $d$.

Thus every diagonalizable abelian subgroup $A$ of $\operatorname{Stab}(\varphi)$ will be cyclic of size dividing $d-1, d$, or $d+1$. Furthermore, the only non-diagonalizable element commuting with $A$ can be the matrix $M$ corresponding to the automorphism permuting $x_{0}$ and $x_{1}$; we have $M^{-1}=M$ and $M A M=A$ in $\operatorname{PGL}(2)$ if and only if $\frac{a_{1}}{a_{0}}=\frac{a_{0}}{a_{1}}$, or, equivalently, $a_{i}= \pm 1$ for $i=0,1$. In other words, the only possible non-diagonalizable abelian subgroup $A$ is $\mathbb{Z} / 2 \mathbb{Z} \times \mathbb{Z} / 2 \mathbb{Z}$.

Now, the only finite subgroups of PGL(2) are, up to conjugation, cyclic, dihedral, tetrahedral, octahedral, or icosahedral [12]. The last three groups are of order at most 60; only the first two are infinite families. Since the largest abelian subgroup of the dihedral group of order $2 k$ is of order $k$, we see that for large $d$, the order of $\operatorname{Stab}(\varphi)$ is bounded by $2(d+1)$.

We conclude this section with a remark that $\mathrm{M}_{d}^{n}(k)$, consisting of all $k$-rational points in $\mathrm{M}_{d}^{n}(\bar{k})$, is not the same as the quotient $\operatorname{Hom}_{d}^{n}(k) / \operatorname{PGL}(n+1, k)$. The latter parametrizes morphisms of $\mathbb{P}_{k}^{n}$ up to conjugation defined over $k$, the former up to 
conjugation defined over $\bar{k}$. There exist maps defined over $k$ which are conjugate over $\bar{k}$ but not over $k$ itself. For examples, see [13] and $\S \S 4.7-4.10$ of [14].

\section{Rationality of $\mathrm{M}_{d}$}

In this section, we show that when $n=1$, the variety $\mathrm{M}_{d}=\mathrm{M}_{d}^{1}$ is rational. This partly generalizes Silverman's result in $\left[13\right.$, that $\mathrm{M}_{2}=\mathbb{A}^{2}$ over $\mathbb{Z}$. We do so by parametrizing fixed points of $\varphi$. The fixed point set of $\varphi, \operatorname{Fix}(\varphi)$, is the intersection of two curves in $\mathbb{P}^{1} \times \mathbb{P}^{1}$, the graph $\Gamma_{\varphi}$ and the diagonal embedding $\Delta$. As $\Delta$ is irreducible and not contained in $\Gamma_{\varphi}$ for $d>1$, this is a proper intersection of divisors of type $(1,1)$ and $(d, 1)$, so it has $d+1$ points, counting multiplicity. We have:

Theorem 4.1. $\mathrm{M}_{d}$ is birational to the total space of a rank-d vector bundle on $\mathrm{M}_{0, d+1}$, the space of unmarked $d+1$ points on $\mathbb{P}^{1}$. Since $\mathrm{M}_{0, d+1}$ is rational, it follows that $\mathrm{M}_{d}$ is rational.

Proof. We explicitly write $\varphi(x: y)=(p: q)$ where $p(x, y)=a_{d} x^{d}+\ldots+a_{0} y^{d}$ and $q(x, y)=b_{d} x^{d}+\ldots+b_{0} y^{d}$. The fixed points of $\varphi$ are those for which $(p: q)=(x: y)$, which are the roots of the homogeneous degree- $d+1$ polynomial $p y-q x$. The polynomial $p y-q x$ induces a map from $\operatorname{Rat}_{d}$ to $\left(\mathbb{P}^{1}\right)^{d+1} / S_{d+1}$ where $S_{d+1}$ acts by permutation of the factors. We will call this map Fix. We use the following lemma:

Lemma 4.2. The map Fix is surjective, and has rational fibers.

Proof. A point $(x: y)$ is fixed if and only if we have $p y=q x$, i.e. $a_{d} x^{d} y+\ldots+a_{0} y^{d+1}=$ $b_{d} x^{d+1}+\ldots+b_{0} x y^{d}$. This is a homogeneous linear condition in the coefficients of $\varphi$, and we have $d+1$ such conditions compared with $2 d+2$ variables. From elementary linear algebra, we have a solution space of linear dimension $d+1$, or projective dimension $d$. It is a linear subvariety of $\mathbb{P}^{2 d+1}$, so it is rational.

We can also show that this dimension- $d$ space will not be contained in the resultant locus. We fix a set of fixed points and write $r$ for the polynomial having those fixed points as roots. We need to show $r$ is of the form $p y-q x$ for some $p$ and $q$ sharing no common root. By conjugating, we may assume neither $(0: 1)$ nor $(1: 0)$ is a root of $r$, so that it has a nonzero $x^{d+1}$ coefficient, which we may take to be 1 , and a nonzero $y^{d+1}$ coefficient. Now we let $q=-x^{d}$ so that $r+q x$ is divisible by $y$, yielding $p=\frac{r+q x}{y}$. Now $r+q x$ has a nonzero $y^{d+1}$ coefficient, so $p$ has a nonzero $y^{d}$ coefficient; therefore, $p$ does not have $(0: 1)$ as a root, so it shares no root with $y$.

Now, Fix descends to a rational map Fix' $\mathrm{M}_{d} \rightarrow\left(\mathbb{P}^{1}\right)^{d+1} / S_{d+1} \operatorname{PGL}(2)$ where PGL(2) acts diagonally; we are restricting to the open set of $\mathrm{M}_{d}$ whose fixed points are in the stable space of the action of $\operatorname{PGL}(2)$ on $\left(\mathbb{P}^{1}\right)^{d+1} / S_{d+1}$. With this restriction, the image is $\mathrm{M}_{0, d+1}$, so it suffices to show the general fiber of $\mathrm{Fix}^{\prime}$ is rational. Lemma 4.2 says that the fiber of Fix is rational, so it suffices to show that the automorphism group of the general point in $\left(\mathbb{P}^{1}\right)^{d+1} / S_{d+1}$ is small enough that the quotient of the fiber by it is still rational. Using Noether's problem [9] [11], we will show a stabilizer of size 4 or 6 is small enough. 
Lemma 4.3. Let $d>1$. The automorphism group of a general configuration of $d+1$ unmarked points in $\mathbb{P}^{1}$ is trivial, unless $d=2$, in which case it is $S_{3}$, or $d=3$, in which case it is $\mathbb{Z} / 2 \mathbb{Z} \times \mathbb{Z} / 2 \mathbb{Z}$.

Proof. We will use inhomogeneous coordinates. For $d=2$, we can conjugate the three points to be $0,1, \infty$; the set is then stabilized by every permutation in $S_{3}$, so it has size 6 . For $d>3$, we will show that the stabilizer is generically trivial, and on the way show that for $d=3$ the stabilizer is generically of order 4 , consisting of all elements in $S_{4}$ of cycle type $(2,2)$. This will be enough to prove the theorem.

First, note that if a $(d+1)$-cycle stabilizes the set of points, then by conjugation we may assume it sends 0 to 1,1 to $\lambda, \mu$ to $\infty$, and $\infty$ to 0 . The cycle, regarded as an element of PGL(2), is of the form $\frac{a x+b}{c x+e}$; then $\frac{b}{e}=1, a=0, \frac{a+b}{c+e}=\lambda$, and $c \mu+e=0$. These equations together imply that $\lambda=\frac{e}{c+e}=\frac{e}{e-\frac{e}{\mu}}=\frac{\mu}{\mu-1}$. For a generic choice of $\mu, \lambda$, this can never happen, so no $(d+1)$-cycle is in the stabilizer. This remains true for $d=3$, in which case we are forced to have $\lambda=\mu$, since generically $\lambda \neq \frac{\lambda}{\lambda-1}$.

Observe that if an automorphism of cycle type $\left(c_{1}, \ldots, c_{k}\right)$ stabilizes the set, then each subset corresponding to the $i$ th cycle is stabilized by a $c_{i}$-cycle. Therefore, the above discussion shows that no cycle of length 4 or more stabilizes a generic set. We have reduced to the case when all cycles are of size 1, 2, or 3 . Now, if we have a stabilizing automorphism which includes a 3-cycle, we may conjugate the 3 -cycle to be $\left(\begin{array}{ll}0 & 1\end{array}\right)$, forcing it to act on $\mathbb{P}^{1}$ as $\frac{1}{1-x}$. Generically, if $\lambda$ is a fourth point, none of the points in the set (including $\lambda$ ) will be $\frac{1}{1-\lambda}$. We are left with cycles of size 1 or 2 . If we have a stabilizing automorphism with two 2-cycles, then up to conjugation we may assume the element acts on four points as $(0 \infty)(1 \lambda)$, so that it maps $x$ to $\frac{\lambda}{x}$. If $d=3$ then this will stabilize the set regardless of what $\lambda$ is. If $d>3$ then we have an additional point $\mu$, and generically $\frac{\lambda}{\mu}$ will not be in our set.

We are left with automorphisms that act as single 2 -cycles, fixing $d-1$ points. For $d \geq 4$, they will fix 3 points and therefore act trivially. For $d=3$, we may assume by conjugation that the element acts as $\left(\begin{array}{ll}0 & 1\end{array}\right)$ and fixes $\infty$; this forces it to be the automorphism $1-x$, which generically does not fix $\lambda$. This leaves us with automorphisms consisting only of 1-cycles, i.e. the identity.

We will return to Noether's problem now. Let us work over a fixed field $k$. Recall [9] that if $K=k\left(x_{1}, \ldots, x_{m}\right)$ is a purely transcendental field, and $G$ is a finite group of size $2,3,4$, or 6 permuting the $x_{i}$ 's, then $K^{G}$ is purely transcendental as well. In particular, if $R$ is the graded $k$-algebra $k\left[x_{1}, \ldots, x_{m}\right]$, and $G$ acts on it by permutation of the $x_{i}$ 's, then $\operatorname{Proj} R^{G}$ is rational. We will show this to be the case when $R$ is the fiber of Fix in the $d=2$ and $d=3$ cases, by finding an orbit $y_{1}, \ldots, y_{m}$ generating $R$ over $k$.

When $d=2$, we have a 2-dimensional fiber. Explicitly, we have six homogeneous variables $a_{i}, b_{i}, 0 \leq i \leq 2$, on which the automorphism group PGL(2) acts linearly. The fiber we are interested in consists of maps fixing the points $0,1, \infty$, corresponding to the linear conditions $a_{0}=0, a_{0}+a_{1}+a_{2}=b_{0}+b_{1}+b_{2}, b_{2}=0$, respectively. The values 
of $a_{2}, a_{1}, b_{0}$ uniquely determine that of $b_{1}$, so we may write the fiber as $\operatorname{Proj} k\left[a_{2}, a_{1}, b_{0}\right]$. The group $S_{3}$ acts linearly and faithfully on the $k$-vector space spanned by $a_{2}, a_{1}, b_{0}$. Let us consider the action of the automorphism $(0 \infty)=\frac{1}{x}$ :

$$
\begin{gathered}
\varphi(x)=\frac{a_{2} x^{2}+a_{1} x}{b_{1} x+b_{0}} \\
\frac{1}{\varphi\left(\frac{1}{x}\right)}=\frac{b_{0} x^{2}+b_{1} x}{a_{1} x+a_{2}} \\
a_{2} \mapsto b_{0} \\
a_{1} \mapsto b_{1}=a_{2}+a_{1}-b_{0} \\
b_{0} \mapsto a_{2}
\end{gathered}
$$

Observe that this automorphism fixes $a_{2}+b_{0}$. Let us also consider the action of the automorphism $\left(\begin{array}{ll}0 & 1\end{array}\right)=1-x$ :

$$
\begin{aligned}
& 1-\varphi(1-x)= 1-\frac{a_{2}(1-x)^{2}+a_{1}(1-x)}{b_{1}(1-x)+b_{0}} \\
&= \frac{-a_{2}(1-x)^{2}+\left(b_{1}-a_{1}\right)(1-x)+b_{0}}{b_{1}(1-x)+b_{0}} \\
& a_{2} \mapsto-a_{2} \\
& a_{1} \mapsto 2 a_{2}+a_{1}-b_{1}=a_{2}+b_{0} \\
& b_{0} \mapsto b_{0}+b_{1}=a_{2}+a_{1}
\end{aligned}
$$

This automorphism does not stabilize $a_{2}+b_{0}$; hence, $a_{2}+b_{0}$ has stabilizer of order 2 , and orbit of size 3 . By repeating the maps $1-x$ and $\frac{1}{x}$, we can compute the orbit as $\left\{a_{2}+b_{0}, a_{1}, a_{2}+a_{1}-b_{0}\right\}$. This generates $R$ as long as char $k \neq 2$. When char $k=2$, the automorphism $1-x$ fixes $a_{2}$, whose orbit is then $\left\{a_{2}, b_{0}, a_{2}+a_{1}\right\}$. In either case, we can construct the action of $S_{3}$ as an action of generators, reducing the quotient to Noether's problem.

When $d=3$, we similarly obtain a 3 -dimensional fiber, fixing the points $0,1, \lambda, \infty$. We obtain the linear conditions $a_{0}=0, b_{3}=0, a_{3}+a_{2}+a_{1}=b_{2}+b_{1}+b_{0}, \lambda^{2} a_{3}+$ $\lambda a_{2}+a_{1}=\lambda^{2} b_{2}+\lambda b_{1}+b_{0}$, and we may write $R$ as $k\left[a_{3}, a_{2}, b_{1}, b_{0}\right]$. We look at the automorphism $(0 \infty)(1 \lambda)=\frac{\lambda}{x}$ :

$$
\begin{gathered}
\varphi(x)=\frac{a_{3} x^{3}+a_{2} x^{2}+a_{1} x}{b_{2} x^{2}+b_{1} x+b_{0}} \\
\frac{\lambda}{\varphi\left(\frac{\lambda}{x}\right)}=\frac{\lambda}{\frac{a_{3} \lambda^{3}+a_{2} x \lambda^{2}+a_{1} x^{2} \lambda}{b_{2} x \lambda^{2}+b_{1} x^{2} \lambda+b_{0} x^{3}}}=\frac{b_{0} x^{3}+b_{1} \lambda x^{2}+b_{2} \lambda^{2} x}{a_{1} x^{2}+a_{2} \lambda x+a_{3} \lambda^{2}} \\
a_{3} \mapsto b_{0} \\
a_{2} \mapsto \lambda b_{1}
\end{gathered}
$$




$$
\begin{gathered}
b_{1} \mapsto \lambda a_{2} \\
b_{0} \mapsto \lambda^{2} a_{3}
\end{gathered}
$$

We may scale down by a factor of $\lambda$ to obtain $\left(\lambda^{-1} b_{0}, b_{1}, a_{2}, \lambda a_{3}\right)$, which is equivalent to picking the representative function $\frac{\sqrt{\lambda}}{\sqrt{\lambda^{-1}} x}$. Let us also consider the action of the automorphism $(0 \lambda)(1 \infty)=\frac{x-\lambda}{x-1}$ :

$$
\varphi\left(\frac{x-\lambda}{x-1}\right)=\frac{a_{3}(x-\lambda)^{3}+a_{2}(x-\lambda)^{2}(x-1)+a_{1}(x-\lambda)(x-1)^{2}}{b_{2}(x-\lambda)^{2}(x-1)+b_{1}(x-\lambda)(x-1)^{2}+b_{0}(x-1)^{3}}
$$

We obtain:

$$
\begin{gathered}
\frac{a_{3}(x-\lambda)^{3}+\left(a_{2}-\lambda b_{2}\right)(x-\lambda)^{2}(x-1)+\left(a_{1}-\lambda b_{1}\right)(x-\lambda)(x-1)^{2}-\lambda b_{0}(x-1)^{3}}{a_{3}(x-\lambda)^{3}+\left(a_{2}-b_{2}\right)(x-\lambda)^{2}(x-1)+\left(a_{1}-b_{1}\right)(x-\lambda)(x-1)^{2}-b_{0}(x-1)^{3}} \\
a_{3} \mapsto a_{3}+a_{2}+a_{1}-\lambda\left(b_{2}+b_{1}+b_{0}\right)
\end{gathered}
$$

We will show the orbit of $a_{3}$ generates $R$. But first, note that $a_{3}+a_{2}+a_{1}=b_{2}+b_{1}+b_{0}$ implies that $a_{1}=b_{2}+b_{1}+b_{0}-a_{2}-a_{3}$, and then $\lambda^{2} a_{3}+\lambda a_{2}+a_{1}=\lambda^{2} b_{2}+\lambda b_{1}+b_{0}$ implies that $\left(\lambda^{2}-1\right) a_{3}+(\lambda-1) a_{2}=\left(\lambda^{2}-1\right) b_{2}+(\lambda-1) b_{1}$, that is, $b_{2}=a_{3}+\frac{a_{2}-b_{1}}{\lambda+1}$.

We have $\frac{x-\lambda}{x-1}$ mapping $a_{3}$ to $a_{3}+a_{2}+a_{1}-\lambda\left(b_{2}+b_{1}+b_{0}\right)=(1-\lambda)\left(b_{2}+b_{1}+b_{0}\right)=(1-$ $\lambda)\left(a_{3}+b_{0}+\frac{a_{2}+\lambda b_{1}}{\lambda+1}\right)$. If we then apply the map $\frac{\lambda}{x}$, we obtain $(1-\lambda)\left(\lambda^{-1} b_{0}+\lambda b_{3}+\frac{b_{1}+\lambda a_{2}}{\lambda+1}\right)$. The orbit is, up to scaling, $\left\{a_{3}, b_{0}, a_{3}+b_{0}+\frac{a_{2}+\lambda b_{1}}{\lambda+1}, \lambda^{-1} b_{0}+\lambda b_{3}+\frac{b_{1}+\lambda a_{2}}{\lambda+1}\right\}$, which generates $R$. Again, we apply Noether's problem and obtain a rational quotient, as desired.

Unfortunately, this proof does not seem to generalize to $\mathrm{M}_{d}^{n}$. Although Lemma 4.3 is true for all $n, d>1$, there are two significant obstructions. First, the dimension of the target space of the map Fix will be $n\left(1+d+\ldots+d^{n}\right)$, which is larger than $N_{d}^{n}$ unless $n$ and $d$ are very small. This means that the map will not be surjective, though the fibers are still rational whenever they are nonempty. And second, even for small $n$ and $d$ the base space for the vector bundle is not $\mathrm{M}_{0, d+1}$, which is relatively tame, but rather the space of $1+d+\ldots+d^{n}$ points on $\mathbb{P}^{n}$, a much more complex object. All we can say at this stage is that $\mathrm{M}_{d}^{n}$ is unirational, which follows trivially from the fact that it is covered by $\operatorname{Hom}_{d}^{n}$.

\section{References}

[1] Susan Claire Dancs, Abelian subgroups of finite p-groups, Trans. Amer. Math. Soc. 169 (1972), 489-493. MR MR0308258 (46 \#7372)

[2] Laura DeMarco, Iteration at the boundary of the space of rational maps, Duke Math. J. 130 (2005), no. 1, 169-197. MR MR2176550 (2006k:37130)

[3] (2007), no. 2, 321-355 (electronic). MR MR2276773 (2008c:14021) 
[4] F. S. Macaulay, The algebraic theory of modular systems, Cambridge Mathematical Library, Cambridge University Press, Cambridge, 1994, Revised reprint of the 1916 original, With an introduction by Paul Roberts. MR MR1281612 (95i:13001)

[5] Michelle Manes, Moduli spaces for families of rational maps on $\mathbb{P}^{1}$, arXiv:0902.1813v2, Feb 2009.

[6] G. A. Miller, On the number of abelian subgroups whose order is a power of a prime, Messenger Math. 36 (1906-7), 79-80.

[7] John Milnor, Dynamics in one complex variable, third ed., Annals of Mathematics Studies, vol. 160, Princeton University Press, Princeton, NJ, 2006. MR MR2193309 (2006g:37070)

[8] David Mumford and John Fogarty, Geometric invariant theory, second ed., Ergebnisse der Mathematik und ihrer Grenzgebiete [Results in Mathematics and Related Areas], vol. 34, Springer-Verlag, Berlin, 1982. MR MR719371 (86a:14006)

[9] Emmy Noether, Gleichungen mit vorgeschriebener Gruppe, Math. Ann. 78 (1964), no. 1, 221-229. MR MR1511893

[10] Clayton Petsche, Lucien Szpiro, and Michael Tepper, Isotriviality is equivalent to potential good reduction for endomorphisms of $\mathbb{P}^{n}$ over function fields, arXiv:0806.1364v2, Nov 2008.

[11] David J. Saltman, Noether's problem over an algebraically closed field, Invent. Math. 77 (1984), no. 1, 71-84. MR MR751131 (85m:13006)

[12] Joseph H. Silverman, The field of definition for dynamical systems on $\mathbf{P}^{1}$, Compositio Math. 98 (1995), no. 3, 269-304. MR MR1351830 (96j:11090)

[13] _ The space of rational maps on $\mathbf{P}^{1}$, Duke Math. J. 94 (1998), no. 1, 41-77. MR MR1635900 (2000m:14010)

[14] - The arithmetic of dynamical systems, Graduate Texts in Mathematics, no. 241, Springer-Verlag, New York, 2007.

Alon Levy, Department of Mathematics, Columbia University, New York, NY 10027, USA

EMAIL: LEVY@MATH. COLUMBIA.EDU 